\title{
EXTENSION OF DERIVATIONS IN CONTINUOUS TRANSFORMATION RINGS $\left({ }^{1}\right)$
}

\author{
BY \\ ALEX ROSENBERG AND DANIEL ZELINSKY
}

1. Introduction. If $A$ is a finite-dimensional central simple algebra, any derivation of a semisimple subalgebra $C$ into $A$ may be extended to an inner derivation of $A[4$, p. 102]. Nakayama [7, Theorem 2] has generalized this result to the situation where $A$ and $C$ are simple rings with minimum condition containing the same unit, and the derivation annihilates a simple weakly Galois (cf. definition below) subring $C_{0}$ of $C$. In this paper we extend Nakayama's theorem to the case where $A$ is a continuous transformation ring (i.e. the ring of all continuous linear transformations on a pair of dual spaces), $C$ is a primitive ring with nonzero socle $\left({ }^{2}\right)$ satisfying certain reducibility conditions, and $C_{0}$ is an arbitrary weakly Galois subring of $C$. Under similar hypotheses we prove one further extension theorem where now we do not assume the existence of a weakly Galois subring but instead suppose only that $C$ is a subalgebra over the center of $A$ satisfying a certain finiteness hypothesis.

We are indebted to Professor N. Jacobson for letting us see a manuscript of a book on the theory of rings to be published in the Colloquium series of the American Mathematical Society.

2. Completely primitive rings. Throughout this paper we are concerned with an additive abelian group $M$ and certain rings of endomorphisms on it -i.e. our rings are all subrings of the ring $E$ of all endomorphisms of $M$.

By a derivation $\delta$ of a ring $R$ into a ring $S$ containing $R$ we mean an additive mapping of $R$ into $S$, such that for any $a, b$ in $R,(a b) \delta=(a \delta) b+a(b \delta)$. It is well known that the mapping $a \rightarrow[a, s]=a s-s a$ for some $s$ in $S$ is such a derivation. When $R=S$ this is called the inner derivation by $s$.

In the book on ring theory referred to above Jacobson proves the following lemma by exhibiting the connection between derivations and module extensions. The proof below is a direct one.

Lemma 1. Let $C$ be a primitive subring of $E$ with nonzero socle $S$, such that $\left({ }^{3}\right)$ $M S=M$. Then any derivation $\delta$ of $C$ into $E$ can be extended to an inner one on $E$.

Presented to the Society, September 3, 1954; received by the editors September 11, 1954.

(1) This paper was written while the authors held a grant from the National Science Foundation.

(2) For a discussion of continuous transformation rings and primitive rings with nonzero socles (P.M.I. rings) see $[11, \S \S 1,2]$.

(3) This condition is equivalent to the complete reducibility of $M$ as a $C$-module $[2, \mathrm{p} .158]$ and $[11,(2.7)]$. 
Proof. Let $e$ be any primitive idempotent of $C$, i.e. $e C$ is a minimal right ideal of $C$. As in the proofs of [3, Theorem 4.1] [6, Theorem 9] we subtract an inner derivation of $E$ from $\delta$ to obtain a derivation $\delta_{1}$ of $C$ into $E$ which vanishes on $e$ : since $e^{2}=e,(e \delta) e+e(e \delta)=e \delta$ and so $e(e \delta) e=0$. If $a_{1}=(e \delta) e-e(e \delta)$ then $\left[e, a_{1}\right]=-e(e \delta)-(e \delta) e=-e \delta$. Thus $c \delta_{1}=c \delta+\left[c, a_{1}\right]$ is a derivation vanishing on $e$.

Since $M S=M, M$ is a completely reducible $C$-module $\left({ }^{3}\right), M=\sum_{\oplus} M_{\alpha}$ where the $M_{\alpha}$ are faithful irreducible $C$-modules. Since $M_{\alpha} e C \neq 00$ we may write $M_{\alpha}=x_{\alpha} e C$ for a certain element $x_{\alpha}$ in $M_{\alpha}$. Since $e C$ is a minimal right ideal, $x_{\alpha} e c=0$ only if $e c=0$. Hence if we let $x_{\alpha} e c a_{2}=x_{\alpha}(e c) \delta_{1}=x_{\alpha} e\left(c \delta_{1}\right)$ for every $\alpha$, then $a_{2}$ is a well defined element of $E$. Now for any $b$ in $C$,

$$
x_{\alpha} e c\left[b, a_{2}\right]=x_{\alpha} e c b a_{2}-x_{\alpha} e c a_{2} b=x_{\alpha} e\left((c b) \delta_{1}-\left(c \delta_{1}\right) b\right)=x_{\alpha} e c\left(b \delta_{1}\right)
$$

so that $b \delta_{1}=\left[b, a_{2}\right]$ and $b \delta=\left[b, a_{1}-a_{2}\right]$.

That the assumption $M S=M$ is essential in the above lemma can be seen from the following example (cf. [9, pp. 129-130]): Let $M$ be a vector space of countable dimension over a field $Z$ and let $\left\{x_{i}\right\}(i=0,1,2, \cdots)$ be a basis of $M$ over $Z$. We consider the algebra $C$ of linear transformations of $M$ spanned over $Z$ by the linear transformations $e_{i j}(i, j=1,2, \cdots)$ defined thus:

$$
x_{0} e_{i j}=x_{j}, i \text { odd; } \quad x_{0} e_{i j}=0, i \text { even; } \quad x_{n} e_{i j}=\delta_{n i} x_{j}, \quad n>0 .
$$

Clearly $C$ is isomorphic to the algebra of linear transformations it induces on the space spanned by $x_{1}, x_{2}, \ldots$ namely, the algebra of all finite matrices, which is known to be primitive and is its own socle $S[5$, p. 18]. Hence $M=M S \oplus x_{0} z$. We now consider the linear transformations $e_{i j}^{\prime}$ given by

$$
x_{0} e_{i j}^{\prime}=x_{j} ; \quad x_{n} e_{i j}^{\prime}=0, \quad n>0 .
$$

It is readily verified that $e_{i j}^{\prime} e_{p q}+e_{i j} e_{p q}^{\prime}=\delta_{j p} e_{i q}^{\prime}$ so that the mapping $\sum e_{i j} \alpha_{i j}$ $\rightarrow \sum e_{i j}^{\prime} \alpha_{i j}, \alpha_{i j}$ in $Z$, is a derivation of $C$ into the algebra of linear transformations of $M$. But for any endomorphism $a$ of $M, x_{0} a=\sum_{0}^{m} x_{h} \alpha_{h}$, so that if $i$ is even and $i>m, x_{0}\left(e_{i j} a-a e_{i j}\right)=0 \neq x_{0} e_{i j}^{\prime}$. Thus the derivation cannot be extended to an inner one in this case.

In his book Jacobson uses Lemma 1 to prove the next two theorems. Since they have appeared nowhere else and we shall need them in proving Theorems 3 and 4 , we reproduce them here.

These theorems concern subrings of the centralizer $\mathcal{L}(M)$ in $E$ of a division subring $D$ of $E$. In Theorem 1 the ring of constants contains a ring $C_{0}$ which is assumed to be weakly Galois in the sense of Dieudonne [2] and Nakayama [7]: the centralizer of $C_{0}$ in $E$ is spanned over $D$ by semilinear transformations.

THEOREM 1. Let $\mathcal{L}(M)$ be the ring of all linear transformations on a vector space $M$ over a division ring $D$. Let $C$ be a primitive subring with nonzero socle 
$S$ such that $M S=M$. Suppose that $C$ contains a weakly Galois subring $C_{0}$. Then any derivation $\delta$ of $C$ into $\mathcal{L}(M)$ annihilating $C_{0}$ can be extended to an inner one on $\mathcal{L}(M)$.

Proof. By Lemma 1 there is an endomorphism $a$ of $M$ such that $c \delta=[c, a]$. Since $c_{0} \delta=0$ for each $c_{0}$ in $C_{0}, a=\sum t_{i} \alpha_{i}, \alpha_{i}$ in $D, t_{i}$ semilinear transformations of $M$. Hence, for each $c$ in $C, c \delta=[c, a]=\sum\left[c, t_{i}\right] \alpha_{i}$ is a linear transformation. Now $\left[c, t_{i}\right]$ is a semilinear transformation with the same automorphism of $D$ as $t_{i}$ so that by Lemme $2 \mathrm{~b}$ of [2] $c \delta$ is also equal to $\sum\left[c, t_{i}\right] \alpha_{i}$ summed only over those $i$ for which $t_{i}$ is associated with an inner automorphism of $D$. But then by multiplying the $t_{i}$ by appropriate elements of $D$ they become linear so that we may write $c \delta=\sum\left[c, t_{i}\right] \beta_{i}, \beta_{i}$ in $D, t_{i}$ in $\mathcal{L}(M)$. If we now write the $\beta_{i}$ in terms of a basis $\left\{\gamma_{j}\right\}$ of $D$ over its center $Z$ with $\gamma_{1}=1$, we obtain $c \delta=\sum\left[c, s_{j}\right] \gamma_{j}$ with $s_{j}$ in $\mathcal{L}(M)$. Since $\mathcal{L}(M) D$ is isomorphic to $\mathcal{L}(M) \otimes_{z} D$ [2, Théorème 3], $\left\{\gamma_{j}\right\}$ is a basis of $\mathcal{L}(M) D$ over $\mathcal{L}(M)$, so that $c \delta=\left[c, s_{1}\right]$.

THEOREM 2. Let $\mathcal{L}(M)$ be the ring of all linear transformations on a vector space $M$ over a division ring $D$ with center $Z$. Let $B$ be a finite-dimensional simple subalgebra of $\mathcal{L}(M)$ over $Z$ containing the unit of $\mathcal{L}(M)$. Then any derivation $\delta$ of $B$ into $\mathcal{L}(M)$ which is $Z$-linear (i.e. $Z \delta=0$ ) can be extended to an inner derivation of $\mathcal{L}(M)$.

Proof. The ring $B D$ of endomorphisms of $M$ is isomorphic to $B \otimes_{z} D$ $[1$, Theorem 8$]$ and so is a simple ring with minimum condition [1, Corollary, p. 98 and Theorem 9]. Extend $\delta$ to a derivation of $B D$ into $E$ by setting $D \delta=0$. The result then follows immediately from Lemma 1 .

Added in proof (July 22, 1955). We have recently exhibited Lemma 1 and Theorem 2 as special cases of analogous results on higher cohomology groups.

3. Continuous transformation rings. We shall next prove an analog (Theorem 3) of Theorem 1 in the case where $\mathcal{L}(M)$ is replaced by a continuous transformation ring $A=\mathcal{L}(M, N)$. A corresponding extension of Theorem 2 is already a special case of Hochschild's result [3, Theorem 4.1] except when $B$ is inseparable over $Z$. We conjecture the extended theorem is true without separability hypotheses but we have no proof.

We first state a lemma which is probably well known.

Lemma 2. Let $(M, N)$ be a pair of dual spaces over a division ring $D$. If $p$ is an endomorphism of $M$ such that there is an adjoint endomorphism $p^{*}$ of $N$ with $(x p, f)=\left(x, f p^{*}\right)$ for all $x$ in $M$ and all $f$ in $N$, then $p$ is in $\mathcal{L}(M, N)$.

Proof. For every $f$ in $N$ and $\alpha$ in $D,((x \alpha) p, f)=\left(x \alpha, f p^{*}\right)=\alpha\left(x, f p^{*}\right)$ $=\alpha(x p, f)=((x p) \alpha, f)$ so that $p$ is a linear transformation. But every linear transformation with an adjoint is continuous.

Theorem 3. Let $A=\mathcal{L}(M, N)$ be a continuous transformation ring and let 
$C$ be a primitive subring of $A$ with nonzero socle $S$. Suppose( $\left.{ }^{4}\right)$ that $M S=M$, $N S^{*}=N, S$ is contained in the socle of $A$, and $C$ contains a weakly Galois subring $C_{0}$ of $A$. (Note that the semilinear transformations spanning the centralizer of $C_{0}$ need not be continuous.) Then any derivation $\delta$ of $C$ into $A$ which vanishes on $C_{0}$ can be extended to an inner one on $A$.

Proof. We first think of $C$ as a subring of $\mathcal{L}(M)$, the ring of all linear transformations of $M$ over $D$. Then Theorem 1 insures the existence of a linear transformation $t$ such that $c \delta=[c, t]$. Now let $e$ be any primitive idempotent of $C$. Just as in the proof of Lemma 1, we find an element $a_{1}$ of $A$ such that $\left[e, t-a_{1}\right]=0$ and we let $\left[c, t-a_{1}\right]=c \delta_{1}$ so that $c \delta_{1}=[c, s]$ with $s$ a linear transformation of $M$ such that $e s=s e$. Now for any $c$ in $C$, $(e c e) \delta_{1}=e\left(c \delta_{1}\right) e=e c s e$ -esce $=[e c e, e s e]$. Since $S$ is in the socle of $A, M e$ is a finite-dimensional space and so $[11,(3.20)]$ assures us that $e A e$ induces all linear transformations on $M e$. Thus there is an element $a_{2}$ in $e A e$ such that $a_{2}=e s e$. We now define a derivation of $C$ into $A$ by $c \delta_{2}=c \delta_{1}-\left[c, a_{2}\right]$, so that $(e C e) \delta_{2}=0$. As in the proof of Lemma 1, we write $M=\sum_{\oplus} x_{\alpha} e C$ and $N=\sum_{\oplus} f_{\beta} e^{*} C^{*}$ for irreducible $C$-modules $x_{\alpha} e C$ and irreducible $C^{*}$-modules $f_{\beta} e^{*} C^{*}$. As before we define an endomorphism $a_{3}$ of $M$ by $x_{\alpha} e c a_{3}=x_{\alpha} e\left(c \delta_{2}\right)$, so that, for any $b$ in $C,\left[b, a_{3}\right]=b \delta_{2}$. If we define $a_{3}{ }^{*}$ by $f_{\beta} e^{*} d^{*} a_{3}{ }^{*}=-f_{\beta} e^{*}\left(d \delta_{2}\right)^{*}$, we have

$$
\begin{aligned}
\left(x_{\alpha} e c a_{3}, f_{\beta} e^{*} d^{*}\right) & =\left(x_{\alpha} e\left(c \delta_{2}\right), f_{\beta} e^{*} d^{*}\right)=\left(x_{\alpha} e\left(c \delta_{2}\right) d e, f_{\beta}\right) \\
& =-\left(x_{\alpha} e c\left(d \delta_{2}\right) e, f_{\beta}\right)=\left(x_{\alpha} e c, f_{\beta} e^{*} d^{*} a_{3}{ }^{*}\right) .
\end{aligned}
$$

Hence by Lemma $2, a_{3}$ is in $A$ and $c \delta=\left[c, a_{1}+a_{2}+a_{3}\right]$.

If in Theorem 3 we are willing to put further hypotheses on $C_{0}$, several of the hypotheses on $C$ can be omitted. For example, if the centralizer of $C_{0}$ is spanned by continuous semilinear transformations, then we need only assume that $C$ is primitive and $M S=M$, for here the proof of Theorem 1 applies verbatim. Second, if $C_{0}$ is assumed to satisfy all the conditions imposed on $C$ in Theorem 3 as well as being weakly Galois, then by a slight modification of [11, Proposition 3], $C$ also satisfies the hypotheses of Theorem 3 and so Theorem 3 is true, assuming only that $C$ is primitive with nonzero socle. In fact, under these hypotheses the centralizer of $C_{0}$ is spanned by continuous semilinear transformations $[11,(3.14)]$. Third, if we assume $C_{0}$ is a Galois subring of $A[11, \S 5]$, then our second remark applies.

Since our conditions on $C$ are automatic when $A$ and $C$ are simple with minimum condition and with the same unit, Theorems 1 and 3 are generalizations of Nakayama's theorem [7, Theorem 2]. The continuous transformation ring $A$ in Theorem 3 cannot be replaced by an arbitrary primitive ring with nonzero socle; for example, replacing $A$ by its own socle invalidates the theorem, i.e. the derivation can of course still be extended to an inner one of $\mathcal{L}(M, N)$ but this derivation need not be an inner one on $\mathcal{f}(M, N)$.

(4) For the significance of these hypotheses on $C$, cf. [11, footnote 5]. 
The following theorem is a sort of dual of Theorem 2; e.g., if $D=Z$ then every centralizer in $A$ of a finite-dimensional simple subalgebra is a $C$ satisfying the hypotheses of Theorem 4 [10, Corollary to Theorem 1].

TheOREM 4. Let $A=\mathcal{L}(M, N)$ be a continuous transformation ring with center the field $Z$. Let $C$ be a primitive subalgebra of $A$ over $Z$ with nonzero socle $S$. Suppose $M S=M, N S^{*}=N$, and that $S$ is contained in the socle of $A$. Then if the division ring of $C$ is finite-dimensional $\left({ }^{5}\right)$ over $Z$, any $Z$-linear derivation $\delta$ of $C$ into $A$ can be extended to an inner derivation of $A$.

Proof. As before we write $c \delta_{1}=c \delta+\left[c, a_{1}\right]$ with $e \delta_{1}=0$ for some primitive idempotent $e$ of $C$. Thus $\delta_{1}$ induces a derivation of $e C e$ into $e A e$ which is $e Z e$-linear. As in Theorem $3, e A e$ is the ring of all linear transformations on $M e$ with center $e Z e$ and so applying Theorem 2 to the subalgebra $e C e$ of $e A e$ we find an element $a_{2}$ in $e A e$ such that $(e c e) \delta_{1}=\left[e c e, a_{2}\right]$. The rest of the proof is identical with that of Theorem 3 .

If we restrict ourselves to the case where $Z$ is perfect, Hochschild's result [3, Theorem 4.1] guarantees that any derivation of $e C e$ into $e A e$ can be extended to any inner one of $e A e$ requiring only that $e A e$ be an algebra over $e Z e$ containing $e C e$. Thus the assumption that $S$ is in the socle of $A$ is no longer needed and we have proved the

Corollary. If the center of $A$ is perfect, Theorem 4 remains true with the assumption that $S$ be contained in the socle of $A$ deleted.

Finally we show by an example that the assumption that $e C e$ be finite dimensional over $e Z e$ cannot be dropped. Let $Z$ be an arbitrary field of characteristic zero and let $C=Z\{x\}$ be the field of formal power series in one variable over $Z$. For $A$ we take the ring of formal power series over $C$ in a variable $y$ such that $y x=2 x y$. This construction goes back to Hilbert and it is shown in [8, pp. 40-41] that $A$ is a division ring. Direct computation shows the center of $A$ is $Z$ and so all the assumptions of Theorem 4 except the finite-dimensionality of $e C e$ over $e Z e$ are fulfilled (note that here $e=1$ and $M=M e$ is a one-dimensional space over $A$ ). Now let $\delta$ be the derivation of $C$ given by $\left(\sum \alpha_{i} x^{i}\right) \delta=\sum i \alpha_{i} x^{i-1}, \alpha_{i}$ in $Z$. Then $\delta$ cannot be extended to an inner derivation of $A$, for if $x\left(\sum b_{j} y^{j}\right)-\left(\sum b_{j} y^{j}\right) x=x \delta=1$ with $b_{j}$ in $C$, we get $\sum\left(1-2^{j}\right) b_{j} x y^{j}=1$ which is a contradiction.

\section{REFERENCES} 87-102.

1. E. Artin and G. Whaples, The theory of simple rings, Amer. J. Math. vol. 65 (1943) pp.

(5) By the division ring of $C$ we mean the centralizer of $C$ on a faithful irreducible $C$-module. It may be concretely represented as $e C e$ where $e$ is any primitive idempotent in $C$. This $e C e$ is clearly an algebra over the isomorph $e Z e$ of $Z$. The present finite-dimensionality hypothesis can be interpreted as the finite-dimensionality of $e C e$ over $e Z e$. 
2. J. Dieudonné, La therie de Galois des anneaux simples et semisimples, Comment. Math. Helv. vol. 21 (1948) pp. 154-184.

3. G. Hochschild, On the cohomology groups of an associative algebra, Ann. of Math. vol. 46 (1945) pp. 58-67.

4. N. Jacobson, The theory of rings, Mathematical Surveys, No. 2, New York, 1943.

5. - On the theory of primitive rings, Ann. of Math. vol. 48 (1947) pp. 8-21.

6. I. Kaplansky, Modules over operator algebras, Amer. J. Math. vol. 75 (1953) pp. 839-858.

7. T. Nakayama, Derivation and cohomology in simple and other rings. I, Duke Math. J. vol. 19 (1952) pp. 51-54.

8. K. Reidemeister, Grundlagen der Geometrie, Berlin, 1930.

9. A. Rosenberg, Subrings of simple rings with minimal ideals, Trans. Amer. Math. Soc. vol. 73 (1952) pp. 115-138.

10. - Finite-dimensional simple subalgebras of the ring of all continuous linear transformations, Math. Zeit. vol. 61 (1954) pp. 150-159.

11. A. Rosenberg and D. Zelinsky, Galois theory of continuoustransformation rings, Trans. Amer. Math. Soc. vol. 76 (1955) pp. 429-452.

NORTHWESTERN UNIVERSITY,

Evanston, Ill. 